\title{
REPRESENTAÇÕES SOCIAIS DE MULHERES IDOSAS SOBRE O ENVELHECIMENTO
}

\section{SOCIAL REPRESENTATIONS OF ELDERLY WOMEN ABOUT AGING}

\section{REPRESENTACIONES SOCIALES DE LAS MUJERES MAYORES SOBRE EL ENVEJECIMIENTO}

Hanna Gadelha Silva ${ }^{1}$ Jéssica de Menezes Nogueira², Edson Batista dos Santos Junior ${ }^{3}$, Daisy Teresinha Reis Coutinho ${ }^{4}$, Maria Célia de Freitas 5 .

\section{RESUMO}

Objetivo: Apreender as representações sociais de mulheres idosas sobre o envelhecimento. Método: Trata-se de uma pesquisa exploratória, descritiva, qualitativa e fundamentada na Teoria das Representações Sociais. O estudo foi realizado com 10 idosas, utilizando como coleta de dados um Formulário Sociodemográfico e a entrevista em profundidade. O material discursivo foi organizado com o auxílio do software IRAMUTEQ 0.7 e analisado por meio da interpretação da Análise de Similitude. Resultados: O termo não foi o que mais se destacou e apresentou um maior número de conexões com os termos: andar, direito, passear, sair, conversar, amigo, missa, igreja, querer, sentir, pensar, cuidar, olhar, comportamento, dentro-casa, antes, agora, diferença. Desse modo, as idosas ancoraram o termo envelhecimento às perdas funcionais e limitações, relacionadas a atividades que não conseguem mais realizar devido ao envelhecimento. As idosas também ancoraram o envelhecimento principalmente nos aspectos negativos físicos (doença, osteoporose, remédio, cansaço), psicológicos (triste, estresse, medo, sofrer) e sociais (desrespeitar, abandonar, respeitar, criar, filho, neto). Conclusão: Por fim, a Enfermagem pode desenvolver cuidados e ações educativas a partir das demandas sociais identificadas pelas representações sociais das mulheres idosas.

Descritores: Envelhecimento; Idoso; Mulheres; Enfermagem.

\section{ABSTRACT}

Objective: To understand the social representations of elderly women about aging. Method: This is an exploratory, descriptive, qualitative research based on the Theory of Social Representations. The study was carried out with 10 elderly women, using a sociodemographic form and the in-depth interview to collect the data. The discursive material was organized with the IRAMUTEQ 0.7 software and analyzed through the interpretation of the Similitude Analysis. Results: The term "not" was the one that stood out the most and presented a greater number of connections with the terms: walking, straight, riding, going out, talking, friend, mass, church, wanting, feeling, thinking, taking care, looking, behavior, indoors, before, now, difference. Thus, the elderly anchored the term aging to functional losses and limitations, related to activities that they can no longer perform due to aging. The elderly women also anchored aging mainly on the negative physical aspects (disease, osteoporosis, medicine, tiredness), psychological (sad, stress, fear, suffering) and social (disrespecting, abandoning, respecting, raising, child, grandchild). Conclusion: Finally, nursing can develop care and educational actions based on the social demands identified by the social representations of elderly women.

Descriptors: Aging; Aged; Women; Nursing.

\section{RESUMEN}

Objetivo: Comprender las representaciones sociales de las mujeres mayores sobre el envejecimiento. Método: Esta es una investigación exploratoria, descriptiva, cualitativa basada en la Teoría de las Representaciones Sociales. La investigación se realizó con 10 mujeres de edad avanzada, utilizando un formulario sociodemográfico y la entrevista en profundidad para la recolección de los datos. El material discursivo se organizó con el software IRAMUTEQ 0.7 y se analizó mediante la interpretación del análisis de similitud. Resultados: El término "no" fue el que más se destacó y presentó una mayor cantidad de conexiones con los términos: caminar, derecho, dar una volta, salir, hablar, amigo, misa, iglesia, querer, sentir, pensar, cuidar, apariencia, comportamiento, adentro, antes, ahora, diferencia. Por lo tanto, los ancianos anclaron el término envejecimiento a las pérdidas y limitaciones funcionales, relacionadas con actividades que ya no pueden realizar debido al envejecimiento. Las mujeres de edad avanzada también anclaron el envejecimiento principalmente en los aspectos físicos negativos (enfermedad, osteoporosis, medicina, cansancio), psicológico (tristeza, estrés, miedo, sufrimiento) y social (falta de respeto, abandono, respeto, crianza, hijo, nieto). Conclusión: Finalmente, la enfermería puede desarrollar acciones educativas y asistenciales basadas en las demandas sociales identificadas por las representaciones sociales de las mujeres mayores.

Descriptores: Envejecimiento; Anciano; Mujeres; Enfermería.

${ }^{1}$ Enfermeira pela Universidade Estadual do Ceará. ${ }^{2}$ Doutora em Enfermagem pela Escola Anna Nery de Enfermagem da Universidade Federal do Rio de Janeiro. Docente da Universidade Federal do Piauí. ${ }^{3}$ Mestre pela Universidade do Estado do Rio Grande do Norte. Doutorando em Cuidados Clínicos de Enfermagem e Saúde da Universidade Estadual do Ceará. ${ }^{4}$ Mestre em Cuidados Clínicos em Enfermagem e Saúde. Doutoranda em Cuidados Clínicos em Enfermagem e Saúde pela Universidade Estadual do Ceará. ${ }^{5}$ Doutora em Enfermagem Fundamental pela Escola de Enfermagem de Ribeirão Preto da Universidade de São Paulo. Docente da Universidade Estadual do Ceará.

Como citar este artigo:

Silva HG, Nogueira JM, Junior EBS, et al. Representações sociais de mulheres idosas sobre o envelhecimento. Revista de Enfermagem do Centro Oeste Mineiro. 2020;10:e3821. [Access__]; Available in:__. DOI: http://doi.org/10.19175/recom.v10i0.3821 


\section{INTRODUÇÃO}

O envelhecimento populacional, atualmente, é uma realidade mundial e um desafio para a enfermagem e para a sociedade. 0 número de pessoas com mais de 60 anos no Brasil deverá crescer mais rápido do que a média mundial. Enquanto a quantidade de idosos vai duplicar no mundo até o ano de 2050, ela quase triplicará no Brasil(1). Essa nova realidade tem exigido, cada vez mais, transformações na sociedade brasileira para oferecer qualidade de vida aos idosos e construir novos paradigmas para o envelhecimento, além da formulação de planos com o intuito de desfazer os mitos e estereótipos da velhice.

Outro fenômeno demográfico no Brasil é a feminização da velhice, ou seja, a maior concentração de mulheres nesse grupo etário. A razão entre os sexos para a população idosa é de cerca de 0,8 , indicando que existem aproximadamente 80 homens idosos para cada 100 mulheres idosas. Isso ocorre devido à maior taxa de mortalidade masculina ${ }^{(2-3)}$. O predomínio de mulheres idosas é um fenômeno ainda recente e, muitas vezes, suas necessidades permanecem pouco conhecidas.

O envelhecimento é um processo multifacetado que inclui aspectos biológicos, culturais e sociais. Nessa perspectiva, é possível perceber diferenciações de gênero no envelhecimento. Compreende-se que o envelhecer feminino gera uma dicotomia entre perdas, ora com a valorização da experiência e sabedoria, ora pelas perdas e alterações de natureza física, como doenças e mudanças corporais. Diversos fatores acarretam às mulheres riscos e vulnerabilidades na velhice, expondo desigualdades sociais e diferenças de oportunidades que afetam as mulheres idosas, como uma maior probabilidade de trabalho no setor informal, níveis de renda e escolaridade mais baixos, presença de doenças crônicas, incapacidades e isolamento social ${ }^{(4-5)}$.

A compreensão da sociedade sobre o envelhecimento influencia os comportamentos e a forma que os indivíduos encaram a velhice e, até mesmo, o desenvolvimento de políticas públicas e pesquisas científicas ${ }^{(1)}$. Neste sentido, apreender a forma que as idosas percebem o envelhecimento é relevante para compreender seus comportamentos, noções, sentimentos e pensamentos. Além disso, amplia debates que podem proporcionar um novo olhar sobre a velhice com vistas de uma promoção do envelhecimento saudável.

Dessa forma, aponta-se a importância da Enfermagem estudar as demandas da velhice fundamentadas em referenciais teóricos que se preocupam em entender a construção do envelhecimento a partir do senso comum. Assim, a Teoria das Representações Sociais (TRS) foi o referencial escolhido para este estudo por favorecer o conhecimento do significado de fenômenos sociais do grupo e, assim, viabilizar intervenções.

As representações sociais são uma forma de conhecimento socialmente partilhado que constitui uma realidade comum a um grupo. Elas norteiam e estabelecem as condutas, opiniões e crenças dos grupos sociais ${ }^{(6)}$. A TRS propõe que a representação é sempre de alguém (sujeito) sobre algo (objeto). Para um objeto gerar uma representação social deve possuir uma "relevância cultural" ou "espessura social". Desse modo, se torna um objeto de estudo legitimo da ciência, uma vez que apresenta uma relevância para a vida social ${ }^{(7)}$. No caso desse estudo, os sujeitos de interesse especial serão as mulheres idosas e o objeto será o envelhecimento.

A partir das representações sociais das idosas sobre o envelhecimento é possível identificar a cultura, os valores e as crenças que estão por trás dos pensamentos que orientam os seus modos de viver. $O$ enfermeiro possui papel fundamental na prestação de cuidados e atividades educativas frente às demandas cognitivas, sociais e psicológicas do idoso ${ }^{(8)}$. Portanto, compreender a forma que as idosas percebem o envelhecimento favorece futuras ações de cuidado clínico de enfermagem às mulheres idosas e atividades educativas com vista a possibilitar um envelhecimento ativo e saudável.

Diante desse panorama, o presente estudo teve como objetivo apreender as representações sociais de mulheres idosas sobre o envelhecimento.

\section{MÉTODOS}

Trata-se de uma pesquisa qualitativa, fundamentada na Teoria das Representações Sociais. Neste estudo, optou-se por adotar a abordagem processual, que trabalha com a conhecimento sobre a construção das representações pelos processos de objetivação e ancoragem, dois processos sociocognitivos interligados que formam as representações sociais $^{(6)}$. No processo de ancoragem é associado 
ideias desconhecidas em algo já conhecido a partir da história de vida e do pensamento popular, comparando e classificando em categorias. Já a objetivação consiste em reproduzir um conceito em uma imagem, tornando concreto o conhecimento acerca do objeto ${ }^{(7)}$.

A pesquisa foi realizada com idosas que frequentavam um grupo de convivência para idosos em um Centro de Referência de Assistência Social (CRAS), na cidade de Fortaleza, Ceará, tal ação era mediada pelos profissionais ligados ao CRAS. A pesquisa adotou os seguintes critérios de inclusão: ter idade igual ou superior a 60 anos, ser independente, saudável e participante da oficina há pelo menos um mês. Como critério de exclusão: idosas com frequência menor de $80 \%$.

A pesquisadora incialmente compareceu à um encontro da oficina para ambientação, explicando às idosas e às coordenadoras da oficina o objetivo e a metodologia da pesquisa. O grupo era constituído por 10 idosas e, a partir de conversas com as coordenadoras da oficina, foi visto que todas as idosas participavam assiduamente e que não haveria necessidade de exclusões. Também não houve recusas e, portanto, todas as 10 idosas constituíram a amostra da pesquisa.

Os instrumentos utilizados na coleta de dados foram: um formulário sociodemográfico e uma entrevista em profundidade que foram ambos elaborados pela pesquisadora. $O$ formulário sociodemográfico constava perguntas relacionadas a idade, estado civil, escolaridade, ocupação, renda familiar, número de filhos, religião e tempo de participação nas oficinas do CRAS. Para o estudo das representações sociais é necessário conhecer os participantes e o seu contexto, visto que somos influenciados pelos pensamentos e ações dos outros componentes de nosso grupo social.

Utilizou-se a técnica da entrevista em profundidade com 0 uso de um roteiro semiestruturado. Este tipo de estratégia de coleta de dados pode ser conceituada como entrevista em profundidade, pois reforça as possibilidades ilimitadas de expressão do entrevistado sobre o tema proposto e as associações decorrentes, captando os objetos de representações dos sujeitos do estudo ${ }^{(9)}$. Portanto, essa técnica permite o aprofundamento do diálogo e uma interpretação dos discursos dos participantes. 0 instrumento continha a aplicação de questões referentes ao envelhecimento, explorando o que as idosas pensavam e como agiam sobre o envelhecimento.

A pesquisadora compareceu às cinco oficinas e, após o término dos encontros, as idosas eram convidadas para a coleta de dados. A coleta foi realizada entre março e abril de 2018. As oficinas eram realizadas duas vezes por semana e, após cada encontro, eram realizadas as entrevistas de forma individual em sala designada para a pesquisa pelas coordenadoras da oficina, sendo gravadas mediante o consentimento das idosas e logo depois transcritas. O tempo médio de cada entrevista foi de 10 minutos.

A organização dos dados provenientes do formulário sociodemográfico foi feita a partir da tabulação destes no programa Microsoft Office Excel 2016, calculando-se as frequências absolutas e organizando em uma tabela para caracterização da amostra.

O material do discursivo produzido pelas entrevistas de profundidade foi transcrito literalmente e organizado com o auxílio do software IRAMUTEQ (Interface de $\mathrm{R}$ pourles Analyses Multidimensionnelles de Textes et de Questionnaires) 0.7, versão alpha 2, R 3.1.2., que realiza análises estatísticas de textos e tabelas de palavras. A análise foi desenvolvida por meio da interpretação da Análise de Similitude e discussão com a literatura sobre o tema, a qual baseia-se na teoria dos grafos para identificar as coocorrências entre as palavras e indicar a conexidade entre as palavras $^{(10)}$.

A partir da análise do material verbal transcrito, processado pelo IRAMUTEQ e analisado pela pesquisadora, podemos compreender os processos de ancoragem e objetivação dos sujeitos, um grupo de mulheres que falam com propriedade pelas vivências do envelhecimento e da velhice. Assim, foi possível realizar uma aglutinação de contextos que determinam representações compartilhadas entre as idosas, organizando um pensamento social produzido em relação a determinado fenômeno, no caso o envelhecimento. Nas discussões, de acordo com a abordagem processual da TRS, poderá se estabelecer relações sobre o processo de construção da velhice contendo as crenças, valores, desejos específicos e modos de viver das idosas ancoradas na dicotomia de perdas e ganhos peculiares à essa fase do ciclo vital.

A pesquisa foi desenvolvida após a aprovação do Comitê de Ética em Pesquisa da Universidade Estadual do Ceará (UECE) sob 
número do parecer 2.523.087 do dia 02 de março de 2018. Todos os princípios relacionados à ética e à legalidade jurídica da Resolução 466/2012 do Conselho Nacional de Saúde (CNS) foram respeitados, preservando as participantes da pesquisa no que diz respeito a não maleficência, autonomia e justiça.

\section{RESULTADOS E DISCUSSÃO}

A amostra do estudo foi caracterizada por um grupo de 10 mulheres idosas, com média de idade de 75,9 anos, que variou entre 61 e 92 anos. Foi predominante o baixo nível de escolaridade (8), a renda de um salário mínimo (7), a viuvez (5), a religião católica (10), a aposentadoria (8) e a participação nas oficinas há mais de 2 anos (6).

A análise de similitude das respostas das entrevistas gerou a árvore máxima apresentada na Figura 1. O corpus foi formado pelo conjunto de entrevistas, com 87 segmentos de texto que permitem identificar a conexão de 78 termos.

Figura 1 - Árvore máxima gerada pela análise de similitude das respostas das entrevistas.



Fonte: Extraído do software IRAMUTEQ, 2018.

A árvore máxima evidencia que as palavras foram polarizadas em torno de um termo que mais se destacou nos discursos e conferiu maior centralidade às representações sociais, o termo não, localizado no centro com maior número de coocorrências entre as palavras.

Percebe-se que ao léxico não apresenta o maior número de conexões com os termos: andar, direito, passear, sair, conversar, amigo, missa, igreja, querer, sentir, pensar, cuidar, olhar, comportamento, dentro-casa, antes, agora, diferença. Desse modo, as idosas ancoraram o termo envelhecimento às perdas funcionais e limitações, relacionadas a atividades que não conseguem mais realizar devido ao envelhecimento, como passear, andar, sair de casa, ir à missa.

A estrutura da representação social do envelhecimento é revelada de forma subjetiva, numa perspectiva de ganhos (experiência e sabedoria) e perdas (doença, limitação, solidão). Estudo envolvendo as representações sociais de diversas faixas etárias encontraram que as perdas, como as perdas da saúde física ou perdas da rede familiar e social, assumem maior relevância para os idosos ${ }^{(11)}$.

Dentre os outros termos que se conectam ao não, encontra-se doente, medo, cansaço, osteoporose, triste, morrer, tomar-remédio, estressar, só, dificuldade. Ao observar os elementos associados ao termo não é possível 
distinguir alguns subconjuntos de significados. Gente conecta-se a dificuldade, difícil, hoje, vida, continuar, doença, sofrer, ruim, dia, maior.

Tendo em vista um panorama de todos os léxicos apontados pelas idosas, vê-se que, neste estudo, elas ancoraram o envelhecimento tanto nos aspectos negativos físicos (doença, osteoporose, remédio, cansaço), quanto nos psicológicos (triste, estresse, medo, sofrer) e sociais (desrespeitar, abandonar, respeitar, criar, filho, neto).

As representações sociais apontadas nas entrevistas demonstraram que as idosas ancoraram o envelhecimento principalmente por seus aspectos negativos e suas limitações. Muitas delas, descrevem que as condições crônicas diminuem a sua capacidade funcional e seu bemestar. Idosas de um estudo também ressaltaram as atividades que desejariam realizar, mas que não podem devido à idade. $O$ envelhecimento marca $a$ vida das idosas com novas mudanças em que elas precisam se adaptar. Essas mudanças podem reduzir a satisfação das idosas com a vida, como a perda de habilidades, o cansaço, restrições e problemas de saúde ${ }^{(5)}$.

Foram encontrados dados semelhantes em outro estudo, os quais as idosas ancoraram o envelhecimento, muitas vezes, apenas ao aspecto funcional do corpo humano, com associações negativas de limitações e perdas ${ }^{(12)}$. Um estudo descreveu que as pessoas idosas compreendem o conceito de saúde envolvendo autonomia, capacidade funcional e atitudes de enfrentamento, mudança e adaptação às adversidades da vida ${ }^{(13)}$. Isso ressalta o foco nos aspectos funcionais e físicos das representações do envelhecimento. Portanto, as idosas ancoram a ideia de que o envelhecimento acarreta problemas de saúde e que é necessário conviver com eles e tratá-los.

Diante disso, para as idosas, o envelhecimento positivo é aquele que permite para as mulheres a continuidade da execução das tarefas que sempre realizaram. Destacando-se as atividades domésticas e o papel de cuidadora dos seus familiares, papéis presentes ao longo de suas vidas $^{(14)}$.

Além disso, outros aspectos relevantes na estrutura das representações sociais das idosas são as relações sociais e o convívio em sociedade. Observa-se o desvelar de elementos que possuem uma conexão mais forte entre si, possibilitando a formação de alguns grupos de significados: antesbem-tratar-calma-desrespeitar, nunca-respeitar e filho-neto-abandonar-gosto-criar. Durante as entrevistas, foram relatadas situações em que as idosas são bem tratadas pelos familiares, mas, também, desrespeitadas pela sociedade.

Em estudos, o convívio com a família foi identificado pelos idosos como a melhor alternativa na velhice. A família foi ancorada como uma expressão de apoio fundamental e de valorização da afetividade, enquanto o abandono dos filhos e conflitos intergeracionais são destacados como algo indesejado(15-16). Outro estudo encontrou esse desejo do convívio familiar principalmente nos discursos das idosas quando comparado aos idosos, visto que para muitas o principal investimento ao longo da vida foi o familiar, que se manteve na velhice ${ }^{(17)}$.

Também é importante destacar que a representação da sociedade brasileira, apoiada sobre valores tradicionalmente atribuídos à família, ancora a família como uma instituição essencial para a sobrevivência e cuidado das pessoas idosas $^{(16)}$. Um estudo descreveu que as representações sociais de idosos sobre a família ressaltam aspectos como união, companheirismo, apoio, respeito e cuidados. Assim, o ambiente familiar tem a finalidade de acolher e de abrigar. Por outro lado, foi identificado uma relação de dependência com a família, revelando uma possível associação com o estigma da velhice como um período de declínio e incapacidade ${ }^{(18-19)}$.

Ademais, o envelhecimento foi objetificado pelas idosas na figura do idoso, sendo personificado em familiares e amigos, o que é possível observar através dos elementos marido, pai, amigo. Estudo realizado com mulheres adultas e idosas também identificou a objetivação de ambas as faixas etárias nas figuras familiares idosas $^{(15)}$.

Em contrapartida, a ancoragem das idosas diverge das novas perspectivas da velhice focadas no lazer, na felicidade e na liberdade. A sociedade hipervaloriza a juventude, desse modo, a boa velhice é vista como uma extensão da juventude, apresentando estabilidade financeira e tempo para realizar atividades prazerosas, como viajar, passando a ideia que aproxima a velhice de um período de "eternas férias"(20). A pessoa idosa considerada saudável seria, então, aquela ativa e que mantém as suas atividades do cotidiano.

Um estudo encontrou que quanto mais avançada a idade das pesquisadas, mais aspectos positivos apareciam em seus depoimentos. As idosas retratavam a velhice como um momento de realizações, de liberdade e repleto de aspectos 
positivos. Livres de obrigações familiares e sociais, passavam a priorizar os próprios desejos e realizar seus projetos ${ }^{(21)}$.

Já em outro estudo, as idosas relatavam que mais oportunidades de estudo no passado poderiam garantir maiores facilidades na velhice $^{(5)}$, destacando o impacto da baixa escolaridade e das desigualdades socioeconômicas na vida das idosas. É importante ressaltar que diferentes representações sociais podem ser elaboradas sobre um mesmo objeto, como o envelhecimento. Essas diferenças estão associadas às atividades e características culturais de determinados grupos. Portanto, no estudo das representações sociais, é relevante identificar o contexto característico do grupo ${ }^{(22)}$.

Realçamos que as idosas deste estudo são caracterizadas como um grupo de baixa escolaridade e de baixa renda. Percebe-se como o contexto social em que as idosas estão inseridas implica na prevalência de incapacidade funcional nos idosos e, assim, repercute em suas representações rociais. A baixa renda, a baixa escolaridade, a maior faixa etária e o sexo feminino apresentam maiores proporções de incapacidade funcional(23). É essencial $O$ conhecimento aprofundado dessas desigualdades para o levantamento de demandas de cuidado, o desenvolvimento de políticas públicas eficazes e a realização de intervenções com a população idosa.

Um envelhecimento saudável possui como finalidade o aumento da expectativa de uma vida saudável, a manutenção da capacidade funcional e da qualidade de vida, incluindo o bem-estar físico, social e mental. Além disso, permite a participação contínua nas questões sociais, econômicas, culturais, espirituais e civis da sociedade $^{(24)}$. As mulheres idosas se mostram mais ativas nas atividades socioculturais, principalmente em cultos religiosos. Porém, se encontram menos participativas nas atividades físicas de lazer, principalmente as mulheres de idades mais avançadas ${ }^{(25)}$.

Considerando que as idosas do estudo ancoraram $\mathrm{o}$ envelhecimento nas suas incapacidades funcionais e nos seus aspectos negativos, ressalta-se a necessidade de 0 enfermeiro realizar atividades educativas para promover a manutenção da capacidade funcional, da qualidade de vida e da autonomia. Para isso, deve-se planejar ações que orientem a melhora das Atividades de Vida Diária e modos de viver saudáveis. Podemos citar como temáticas relevantes: atividade física, alimentação saudável, melhora da autoestima e autoimagem, ampliação da rede social, melhora da atividade cognitiva e autocuidado para prevenção e controle de doenças e agravos. Para a realização dessas atividades podem ser usadas diferentes abordagens educacionais e metodologias, como: orientações pedagógicas durante a consulta de enfermagem, acompanhamento domiciliar, aconselhamento com dinâmicas motivacionais, grupos educativos, oficinas e estratégias lúdicas ${ }^{(8)}$.

Portanto, a Enfermagem deve buscar estratégias de cuidado pelos processos de educação em saúde com as mulheres e com a sociedade em geral, a fim de identificar possibilidades com vistas a perspectiva do envelhecimento saudável, tornando a velhice uma fase de vida sem estigmas ou preconceitos.

\section{CONSIDERAÇÕES FINAIS}

O estudo possibilitou apreender as representações sociais de mulheres idosas sobre o envelhecimento. Percebemos que as idosas ancoraram o envelhecimento principalmente nas suas limitações e aspectos negativos. Desse modo, o enfermeiro deve estar atento às alterações do processo de envelhecimento e realizar avaliações da capacidade funcional das idosas. Assim, é possível elaborar ações de prevenção de limitações e incapacidades, promovendo o desenvolvimento das Atividades de Vida Diária e Atividades Funcionais da Vida Diária. Destaca-se, ainda, a importância dos grupos de idosos, uma vez que a convivência social auxilia na adaptação às mudanças do processo de envelhecimento.

Nesse contexto, reflete-se sobre o papel do enfermeiro, em conjunto aos demais profissionais de saúde, de realizar intervenções que promovam um envelhecimento saudável das mulheres, tornando possível chegar à velhice com condições de vivê-la plenamente. As representações sociais sugerem informações que vão subsidiar no cuidado de Enfermagem, sobretudo o investimento em ações de educação em saúde com as mulheres e população em geral.

Conclui-se a necessidade de estudos da Enfermagem se voltarem cada vez mais nas nuances do envelhecimento feminino. Espera-se que o estudo possa fortalecer as pesquisas em torno dessa temática e colabore para a reflexão da Enfermagem e da sociedade sobre o envelhecimento feminino, desmistificando preconceitos e fortalecendo a imagem positiva das idosas. 
Ademais, o estudo apresentou como limitação a sua abrangência, visto o número reduzido das participantes e do local específico do estudo. Portanto, outros estudos podem ampliar as possibilidades de investigação com um número maior de participantes e, também, em outras realidades socioculturais.

\section{REFERÊNCIAS}

1- Organização Mundial de Saúde (OMS). Relatório Mundial de Envelhecimento e Saúde. Geneva: OMS; 2015.

2- Instituto Brasileiro de Geografia e Estatística (IBGE). Síntese dos Indicadores Sociais: Uma análise das condições de vida da população brasileira. Rio de Janeiro: IBGE; 2015.

3- Instituto Brasileiro de Geografia e Estatística (IBGE). Mudanças demográficas no Brasil no início do século XXI: Subsídios para as projeções da população. Rio de Janeiro: IBGE; 2015.

4- Dias MJS, Serra J. Mulher, velhice e solidão: Uma tríade contemporânea? Serv Soc Saúde 2018;17(1):9-30.

DOI:

\subsection{6/sss.v0i0.00000000}

5- Almeida AVA, Mafra SCT, Silva EP, Kanso S. A feminização da velhice: Em foco as características socioeconômicas, pessoais e familiares das idosas e o risco social. Textos Contextos 2015;14(1):11531. DOI: $10.15448 / 1677-9509.2015 .1 .19830$

6- Jodelet $D(\mathrm{Org})$. As representações sociais. Rio de Janeiro: UERJ; 2001.

7- Sá CP. A construção do objeto de pesquisa em representações sociais. Rio de Janeiro: EdUerj; 1998.

8- Carvalho KM, Silva CRDT, Figueiredo MLF, Nogueira LT, Andrade EMLR. Intervenções educativas para promoção da saúde do idoso: Revisão integrativa. Acta Paul Enferm. 2018;31(4):446-54. DOI: $10.1590 / 1982-$

\section{2}

9- Silva SA, Herzberg E, Matos LAL. Características da inserção da psicologia nas pesquisas clínicoqualitativas: Uma revisão. Bol Psicol. 2015 [citado em 10 jul 2019]; 65(142):97-111. Disponível em: http://pepsic.bvsalud.org/pdf/bolpsi/v65n142/v6 5n142a09.pdf

10- Camargo BV, Justo AM. Iramuteq: Um software gratuito para análise de dados textuais.

Temas Psicol. 2013;21(2):513-8. DOI: 10.9788/TP2013.2-16

11- Torres TL, Camargo BV, Boulsfield AB, Silva AO. Representações sociais e crenças normativas sobre envelhecimento. Ciênc Saúde Coletiva
2015;20(12):3621-30. DOI: $10.1590 / 1413-$ 812320152012.01042015

12- Silva SPC, Menandro MCS. As representações sociais da saúde e de seus cuidados para homens e mulheres idosos. Saúde Soc. 2014;23(2):626-40. DOI: $10.1590 /$ S0104-12902014000200022

13- Costa JM, Silva GP. Concepções de saúde para idosos acompanhados em ambulatório de geriatria de uma instituição hospitalar. Rev SBPH 2015 [citado em 10 maio 2020]; 18(2):111-28. Disponível em: http://pepsic.bvsalud.org/pdf/rsbph/v18n2/v18n 2a08.pdf

14- Daniel F, Caetano E, Monteiro R, Amaral I. Representações sociais do envelhecimento ativo num olhar genderizado. Anál Psicológica 2016;4(34):353-64. DOI: 10.14417/ap.1020

15- Castro A, Antunes L, Brito AMM, Camargo BV. Representações sociais do envelhecimento e do rejuvenescimento para mulheres que adotam práticas de rejuvenescimento. Psico 2016;47(4):19-30. DOI: $10.15448 / 1980-$ 8623.2016.4.22495

16- Silva DM, Vilela ABA, Oliveira DC, Alves MR. A estrutura da representação social de família para idosos residentes em lares intergeracionais. Rev Enferm UERJ 2015;23(1):21-6. DOI: 10.12957/reueri.2015.8739

17- Zanello V, Silva LC, Henderson, G. Saúde mental, gênero e velhice na instituição geriátrica. Psicol Teor Pesq. 2015;31(4):543-50. DOI: 10.1590/0102-37722015042444543550

18- Araújo LF, Castro JLC, Santos JVO. A família e sua relação com o idoso: Um estudo de representações sociais. Psicol Pesq. 2018;12(2):14-23. 10.24879/2018001200200130

19- Azevedo PAC, Modesto CMS. A (re)organização do núcleo de cuidado familiar diante das repercussões da condição crônica por doença cardiovascular. Saúde Debate 2016;40(110):183-94. DOI: 10.1590/01031104201611014

20- Alexandre SG, Silva HG, Silva VMGN, Rocha VA, Lopes JP, Freitas MC. Concepções de acadêmicos de enfermagem sobre o idoso e a velhice. Rev Enferm UFPE 2016;10(12):4606-11. DOI: 10.5205/reuol.997888449-6-ED1012201623

21- Goldenberg M. Corpo, envelhecimento e felicidade na cultura brasileira. Contemporânea 2011; 9(2):77-85.

DOI:

10.12957/contemporanea.2011.2143 
22- Castro A, Camargo BV. Representações sociais da velhice e do envelhecimento na era digital: Revisão da literatura. Psicol Rev. 2017;23(3):882-900. DOI: 10.5752/P.16789563.2017v23n3p882-900

23- Figueiredo DSTO, Mendes MSF, Malta DC, Melendez GV. Prevalência de incapacidade funcional em idosos: Análise da Pesquisa Nacional de Saúde. Rev RENE 2017;18(4):468-75. DOI: 10.15253/2175-6783.2017000400007

24- Organização Mundial de Saúde (OMS). Envelhecimento ativo: Uma política de saúde. Brasília: Organização Pan-Americana da Saúde; 2005.

25- Sousa NFS, Lima MG, Cesar CLG, Barros MBA. Envelhecimento ativo: Prevalência e diferenças de gênero e idade em estudo de base populacional. Cad Saúde Pública 2018;34(11):1-14. DOI: $\underline{10.1590 / 0102-311 \times 00173317}$

Nota: Este artigo faz parte do Trabalho de Conclusão de Curso intitulado "Representações sociais de mulheres idosas sobre o envelhecimento e a velhice" do Curso de Enfermagem da Universidade Estadual do Ceará.

Recebido em: 02/06/2020

Aprovado em: 04/09/2020

\section{Endereço de correspondência:}

Hanna Gadelha Silva

Universidade Estadual do Ceará. Av. Dr. Silas Munguba, 1700

- Itaperi, CEP: 60714-903, Fortaleza, Ceará, Brasil.

E-mail: hanna.gadelha@aluno.uece.br 